\title{
Nicotinamide Phosphoribosyltransferase Deficiency Potentiates the Antiproliferative Activity of Methotrexate through Enhanced Depletion of Intracellular ATP
}

\author{
Rakesh K. Singh, Leon van Haandel, Daniel P. Heruth, Shui Q. Ye, J. Steven Leeder, \\ Mara L. Becker, and Ryan S. Funk
}

Departments of Pharmacy Practice (R.K.S., R.S.F.) and Pharmacology, Toxicology, and Therapeutics (J.S.L., R.S.F.), University of Kansas Medical Center, Kansas City, Kansas; Divisions of Clinical Pharmacology, Toxicology and Therapeutic Innovation (L.v. H., J.S.L., M.L.B.), Rheumatology (M.L.B.), and Experimental and Translational Genetics (D.P.H., S.Q.Y.), Children's Mercy Kansas City, Kansas City, Missouri; and Department of Biomedical and Health Informatics, University of Missouri Kansas City School of Medicine, Kansas City, Missouri (S.Q.Y.)

Received November 3, 2017; accepted January 26, 2018

\begin{abstract}
Lower plasma nicotinamide phosphoribosyltransferase (NAMPT) levels are associated with improved response to methotrexate (MTX) in patients with juvenile idiopathic arthritis. Cell-based studies confirmed that reduced cellular NAMPT activity potentiates the pharmacologic activity of MTX; however, the mechanism of this interaction has yet to be defined. Therefore, in this study, we investigate the mechanism of enhanced pharmacologic activity of MTX in NAMPT-deficient A549 cells. Small interfering RNA-based silencing of NAMPT expression resulted in a greater than 3-fold increase in sensitivity to MTX $(P<0.005)$ that was completely reversed by supplementation with folinic acid. Despite a $68 \%$ reduction in cellular NAD levels in NAMPT-deficient cells, no change in expression or activity of dihydrofolate reductase was observed and uptake of MTX was not significantly altered. MTX
\end{abstract}

did not potentiate the depletion of cellular NAD levels, but NAMPT-deficient cells had significant elevations in levels of intermediates of de novo purine biosynthesis and were 4-fold more sensitive to depletion of ATP by MTX $(P<0.005)$. Supplementation with hypoxanthine and thymidine completely reversed the antiproliferative activity of MTX in NAMPT-deficient cells and corresponded to repletion of the cellular ATP pool without any effect on NAD levels. Together, these findings demonstrate that increased MTX activity with decreased NAMPT expression is dependent on the antifolate activity of MTX and is driven by enhanced sensitivity to the ATP-depleting effects of MTX. For the first time, these findings provide mechanistic details to explain the increase in pharmacological activity of MTX under conditions of reduced NAMPT activity.

\section{Introduction}

In a previous study by our group, lower plasma concentrations of nicotinamide phosphoribosyltransferase (NAMPT) were associated with improved therapeutic response to the diseasemodifying antirheumatic drug methotrexate (MTX) in patients with juvenile idiopathic arthritis (Funk et al., 2016). Subsequent cell-based studies using small interfering RNA (siRNA)-based gene silencing or the chemical inhibitor of NAMPT, known as FK-866 [2-(E)- $N$-[4-(1-benzoyl-4-piperidinyl)butyl]-3-(3-pyridinyl)2-propenamide hydrochloride] or daporinad, demonstrated that reductions in cellular NAMPT activity resulted in a marked increase in sensitivity to the antiproliferative activity of MTX

This work was supported in part by The University of Kansas and the National Institute of General Medical Sciences [P20GM103549 and P30GM118247].

http://doi.org/10.1124/jpet.117.246199.
(Funk et al., 2016). Although these findings demonstrated that variation in NAMPT activity is associated with variation in MTX response (both in vivo and in vitro), the mechanism of this interaction remains poorly understood and is the focus of this work.

MTX is an antifolate and is the cornerstone of diseasemodifying antirheumatic drug therapy in the treatment of autoimmune arthritis, including juvenile idiopathic arthritis and rheumatoid arthritis. MTX is a potent inhibitor of dihydrofolate reductase (DHFR) and is metabolized intracellularly to form a series of pharmacologically active polyglutamated metabolites that function as direct inhibitors of several folatedependent enzymes (Fairbanks et al., 1999; Kremer, 2004). Through inhibition of the folate-dependent biochemical pathways, MTX causes the inhibition of various downstream onecarbon transfer reactions, including nucleotide and methionine biosynthesis, which are believed to be responsible for its pharmacological activity in the treatment of autoimmune

ABBREVIATIONS: AICART, aminoimidazole carboxamide ribonucleotide transformylase; DHFR, dihydrofolate reductase; DMSO, dimethylsulfoxide; FK-866, 2-(E)-N-[4-(1-benzoyl-4-piperidinyl)butyl]-3-(3-pyridinyl)-2-propenamide hydrochloride; GMX1777, [4-[[N'-[6-(4-chlorophenoxy)hexyl]-N-cyanocarbamimidoyl]amino]pyridin-1-ium-1-yl]methyl 2-[2-[2-(2-methoxyethoxy)ethoxy]ethoxy]ethyl carbonate; HYP, hypoxanthine; IMP, inosine 5'-monophosphate; MTX, methotrexate; NAMPT, nicotinamide phosphoribosyltransferase; PARP, poly(ADP-ribose) polymerase; PBMC, peripheral blood mononuclear cell; PCR, polymerase chain reaction; siRNA, small interfering RNA; THF, tetrahydrofolate; THY, thymidine; ZMP, 5aminoimidazole-4-carboxamide ribonucleotide. 
arthritis (Piper et al., 1983; Smoleńska et al., 1999). Therapeutic response to MTX in the treatment of arthritis continues to be variable, with approximately one in three patients failing to adequately respond to therapy, and has resulted in an effort to understand the mechanistic basis for the observed variation in drug response (Ravelli et al., 1998; Bulatović et al., 2011). Therefore, our observation that variation in NAMPT activity is associated with variation in the pharmacological activity of MTX warrants further investigation to understand the mechanism for this interaction.

NAMPT, also known as pre-B cell colony-enhancing factor or visfatin, functions as the rate-limiting enzyme in the mammalian salvage pathway for intracellular NAD biosynthesis (Samal et al., 1994; Friebe et al., 2011). However, NAMPT is also secreted from cells through an undefined pathway and is found at relatively high concentrations in the plasma, with one study reporting concentrations of $7.2 \pm$ $0.9 \mathrm{ng} / \mathrm{ml}$ in normal healthy adults (Liakos et al., 2016). Although an extracellular receptor for NAMPT is yet to be definitively identified, it is commonly referred to as an adipocytokine and when supplied in its exogenous form has been demonstrated to be important in the regulation of cellular metabolism, inflammation, and immune function (Otero et al., 2006; Moschen et al., 2010; Mirfeizi et al., 2014). NAMPT has been investigated as a plasma biomarker of inflammation and more recently as a potential drug target in the treatment of rheumatoid arthritis (Rongvaux et al., 2008; Evans et al., 2011; Présumey et al., 2013). Inhibition of NAMPT in the collagen-induced arthritis mouse model has demonstrated efficacy similar to that of the anti-tumor necrosis factor $\alpha$ biologic etanercept and has been primarily attributed to the inhibition of intracellular NAD biosynthesis in inflammatory cells (Busso et al., 2008; Evans et al., 2011).

Based on our understanding of the biologic function of NAMPT, it is reasonable to hypothesize that the enhanced sensitivity to MTX observed after inhibition of NAMPT likely results from the depletion of the cellular NAD pool. Previous work investigating the NAMPT inhibitor GMX1777 ([4-[[N'-[6-(4-chlorophenoxy)hexyl]- $N$-cyanocarbamimidoyl] amino]pyridin-1-ium-1-yl]methyl 2-[2-[2-(2-methoxyethoxy) ethoxy]ethoxy] ethyl carbonate), also known as teglarinad chloride, demonstrated that silencing of folate-related genes enhances the cytotoxicity of GMX1777 (Chan et al., 2014). Similarly, Chan et al. (2014) found that the antifolate drug pemetrexed promotes GMX1777 cytotoxicity through the synergistic depletion of cellular NAD resulting from activation of the NAD-consuming DNA repair enzyme poly(ADP-ribose) polymerase (PARP)-1 by pemetrexed. However, in the case of noncytotoxic reduction of cellular NAD through siRNA-based gene silencing of NAMPT, it remains unclear how reductions in NAMPT activity promote the antiproliferative activity of MTX. In this work, we hypothesize that partial depletion of cellular NAD through reduced expression of NAMPT increases the antiproliferative effects of MTX mediated through inhibition of nucleotide biosynthesis and results in the synergistic depletion of cellular ATP.

Here, we investigate the mechanism of increased pharmacological activity of MTX in NAMPT-deficient cells through targeted silencing of NAMPT. Through measurement and supplementation of key metabolic pathways, we derive the biochemical basis through which reduced enzymatic activity of NAMPT promotes the pharmacologic activity of MTX.

\section{Materials and Methods}

Cell Culture. A549 human lung carcinoma cells (catalog no. CCL185) were purchased from American Type Culture Collection (Manassas, VA) and foreskin fibroblasts (AG07095) were provided by Coriell (Camden, NJ). Cells were grown in Dulbecco's modified Eagle's medium (catalog no. 11995-065) supplemented with $10 \%$ fetal bovine serum (catalog no. 03-600-511), $100 \mathrm{U} / \mathrm{ml}$ penicillin, and $100 \mathrm{U} / \mathrm{ml}$ streptomycin (Thermo Fisher Scientific, Waltham, MA). Cells were maintained at $37^{\circ} \mathrm{C}$ in a $5 \% \quad \mathrm{CO}_{2}$ controlled incubator and were passaged every 3 to 4 days at approximately $80 \%$ confluence.

Peripheral Blood Mononuclear Cells. Peripheral blood mononuclear cells (PBMCs) were isolated using density gradient separation (Ficoll-Paque Plus; GE Healthcare, Piscataway, NJ). Blood was diluted with phosphate-buffered saline in equal volume, and $4 \mathrm{ml}$ diluted blood was carefully layered on the top of $3 \mathrm{ml}$ Ficoll in a centrifuge tube to minimize mixing. Samples were centrifuged at $20^{\circ} \mathrm{C}$ for 30 minutes at $400 \mathrm{~g}$. PBMCs were carefully extracted from the centrifuge tube and diluted with phosphate-buffered saline in a $1: 3$ ratio and centrifuged at $100 \mathrm{~g}$ for 10 minutes for washing. Washing was repeated one more time. PBMCs were suspended in RPMI-1640 media supplemented with $10 \%$ fetal bovine serum and incubated for 24 hours to allow monocytes to attach. The next day, the lymphocytes remaining in suspension were seeded at the density of $25 \times 10^{3}$ cells/well in a 96 -well plate with or without $2 \%(\mathrm{v} / \mathrm{v})$ phytohemagglutinin and treated with dimethylsulfoxide (DMSO) or $10 \mathrm{nM}$ MTX for the first 48 hours and then with $0.1 \mathrm{nM}$ FK-866 for the next 72 hours. Cell viability was assessed using the resazurin reduction assay described below.

Cell Viability. For viability studies, cells were seeded at the density of $5 \times 10^{3}$ cells per well in 96 -well clear-bottom black plates (Corning Inc., Corning, NY). With each well containing $100 \mu \mathrm{l}$ growth medium, $20 \mu \mathrm{l} 0.16 \mathrm{mg} / \mathrm{ml}$ resazurin was added and incubated for 4 hours at $37^{\circ} \mathrm{C}$ and $5 \% \mathrm{CO}_{2}$. The plate was protected from light and allowed to cool to room temperature for 10 minutes prior to reading. The fluorescence signal was measured for each sample using a BioTek Cytation 3 (BioTek, Winooski, VT) as described previously (Funk et al., 2016). Protein content was quantified using the Micro BCA Protein Assay Kit (Thermo Fisher Scientific).

NAMPT Inhibition and Drug Treatment. Transfections were performed as described previously (Funk et al., 2016). In brief, in each well of a six-well plate, $250 \mathrm{pmol}$ siRNA and $5 \mu$ l Lipofectamine 2000 (Thermo Fisher Scientific) each in $250 \mu \mathrm{l}$ Opti-MEM reduced serum media (Thermo Fisher Scientific) were mixed at room temperature for 5 minutes before combining with $1.5 \mathrm{ml}$ antibiotic-free growth media containing $2.5 \times 10^{5}$ cells. Volumes and densities were scaled to $5 \times 10^{3}$ cells per well for 96 -well plate assays. After 24 hours, the transfection media were replaced with either normal growth media or normal growth media containing various concentrations of MTX and were maintained for an additional 96 hours under normal culture conditions prior to analysis. For rescue experiments, cells were treated with either MTX alone or MTX with rescue drugs.

Western Blot. Cell lysates were prepared in RIPA buffer (Thermo Fisher Scientific) supplemented with protease and phosphatase inhibitors (Thermo Fisher Scientific). Equal amounts of protein were subjected to SDS-PAGE and then transferred to polyvinylidene membrane (EMD Millipore, Billerica, MA). The membrane was blocked with $5 \%$ nonfat dry milk in phosphate-buffered saline containing $0.1 \%$ Tween 20 at room temperature for 1 hour and was then incubated at $4^{\circ} \mathrm{C}$ overnight with primary antibody. Primary antibodies were rabbit anti-NAMPT (1:4000; generated in house), mouse anti-DHFR (1:750; R\&D Systems, Minneapolis, $\mathrm{MN}$ ), mouse anti-poly(ADP-ribose) clone 10H (1:1000; Sigma-Aldrich, St. Louis, MO), PARP (1:1000; Cell Signaling Technology, Danvers, MA), and glyceraldehyde-3-phosphate dehydrogenase (1:10,000; Santa Cruz Biotechnology, Dallas, TX). After washing three times for 10 minutes with phosphate-buffered saline containing $0.1 \%$ Tween 20, the membrane was incubated with horseradish peroxidase-linked goat anti-rabbit or anti-mouse secondary antibody (Santa Cruz Biotechnology) at room 
A

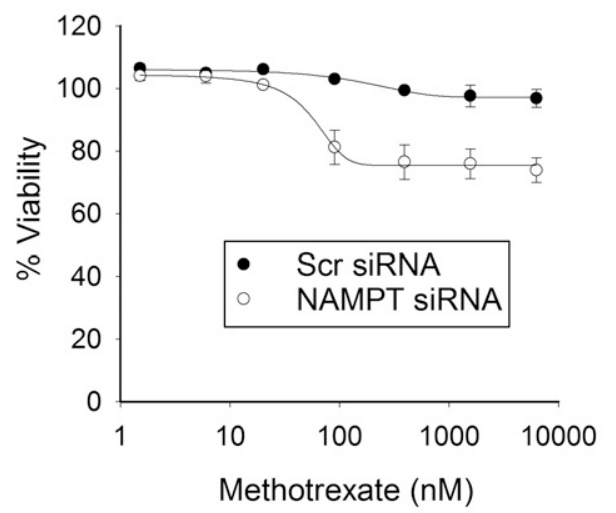

B

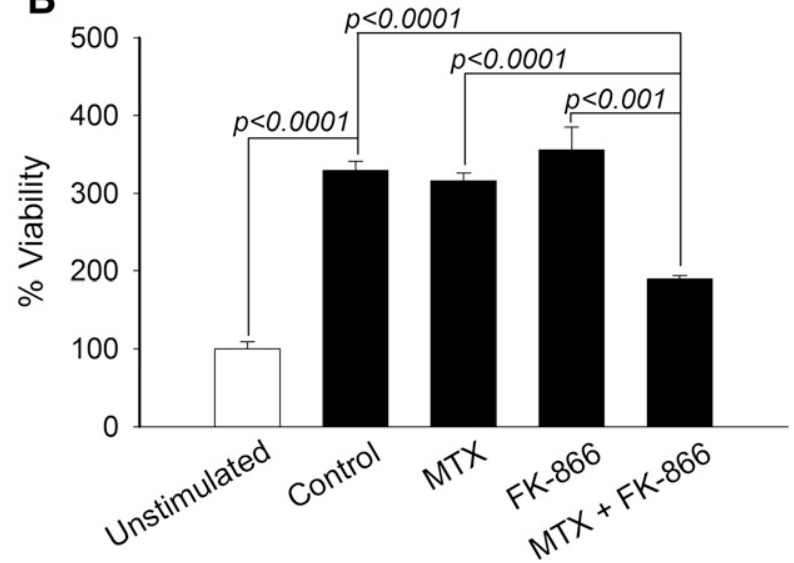

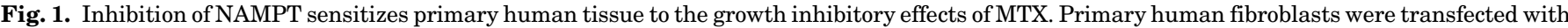

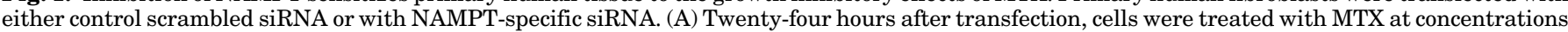

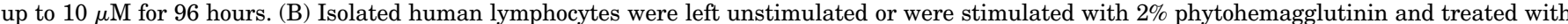

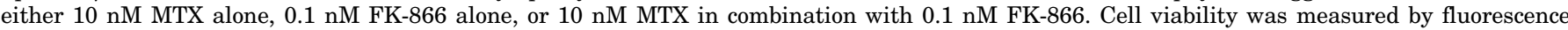

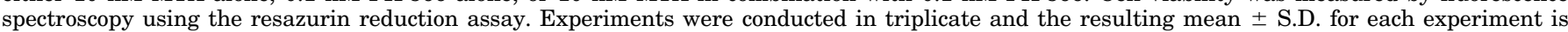
presented. Statistical testing was conducted using $t$ test analysis. Scr, scrambled.

temperature for 1 hour. The blots were then visualized with Pierce ECL Western blot detection reagent (Thermo Fisher Scientific). Band density on Western blot images was used as a measure of assayed protein level.

RNA Isolation, Reverse Transcription, and Real-Time Polymerase Chain Reaction Analysis. Total RNA was isolated from A549 cells transfected with either control or NAMPT siRNA as described in the NAMPT inhibition section with TRIzol reagent (Invitrogen, Carlsbad, CA). One microgram of RNA was used for reverse complementation using the iScript cDNA synthesis kit following the manufacturer's protocol (Bio-Rad, Hercules, CA). Realtime polymerase chain reaction (PCR) was performed with the NAMPT primers sense $5^{\prime}$-tccaccaacacaagcaaagt-3' and antisense $5^{\prime}$-ttcctctgggaatgacaaagccct-3' using the CFX384TM real-time PCR system (Bio-Rad) (Chavan et al., 2015).

Cellular Folate, MTX, and Purine Biosynthesis Intermediate Levels. A549 cells were transfected with either control or NAMPT siRNA as described in the NAMPT inhibition section. Three days later, cells were treated with $1000 \mathrm{nM}$ MTX for 24 hours or were left untreated. Cells were collected by trypsinization and the resulting pellet was flash frozen in liquid nitrogen or immediately processed and analyzed. Cellular folate and MTX levels were analyzed by ultra-performance liquid chromatography tandem mass spectrometry as described previously (Funk et al., 2013, 2014). Cellular levels of 5-aminoimidazole-4-carboxamide ribonucleotide (ZMP) and inosine 5'-monophosphate (IMP) were measured by an additional ultra-performance liquid chromatography-UV assay. Analytes were extracted from cells treated with control or NAMPT siRNA by resuspending the cell pellet in $100 \%$ acetonitrile followed by vortex mixing for 30 seconds and sonication for 5 minutes. Samples were centrifuged at $21,100 \mathrm{~g}$ for 5 minutes and $10 \mu \mathrm{l}$ of the resulting supernatant was injected. Sample separation was conducted on a Waters BEH Amide HILIC chromatography column $(150 \times 3.0 \mathrm{~mm}$ packed with $1.7-\mu \mathrm{m}$ particles; Waters, Milford, MA) using an isocratic method consisting of $33 \% 27 \mathrm{mM}$ potassium dihydrogen phosphate $\mathrm{pH} 4.5$ (mobile phase A) and 77\% acetonitrile (mobile phase B) at a flow rate of $0.9 \mathrm{ml} / \mathrm{min}$. After each run, the column was washed for 2 minutes with a 50/50 mixture of mobile phases A and B. The column was re-equilibrated for 3 minutes under the conditions for isocratic separation prior to the next injection. UV absorption was monitored at 248 and $262 \mathrm{~nm}$ and a five-point calibration curve ranging from 1 to $100 \mu \mathrm{M}$ was constructed for each analyte. Concentrations of ZMP and IMP were determined and normalized based on the average cell count for the samples and were expressed in picomoles per million cells.

NAD and ATP Cellular Levels. Total cellular NAD content was measured using a colorimetric enzyme-linked microplate assay (catalog no. 600480; Cayman Chemical Company, Ann Arbor, MI) and total cellular ATP content was measured using firefly luciferase microplate assays (catalog no. 6016943; PerkinElmer, Waltham, MA). Assays were conducted in a 96-well plate format following the manufacturer's protocol and normalized to total protein.

Drugs and Reagents. Resazurin sodium salt (Thermo Fisher Scientific) was resuspended in $0.16 \mathrm{mg} / \mathrm{ml}$ phosphate-buffered saline, $\mathrm{pH} 7.4$, and passed through a $0.2-\mu \mathrm{m}$ syringe filter. High-performance liquid chromatography purified NAMPT siRNA (sense: 5'-CCACCCAACACAAGCAAAGUUUAUU-3') and scrambled control siRNA (sense: 5'-CCACAACAACAAACGUUGAUCCAUU-3') were custom synthesized (Thermo Fisher Scientific). Thymidine (THY) (catalog no. CAS 50-89-5; Acros Organics, Morris Plains, NJ), hypoxanthine (HYP) (catalog no. 105451) and folinic acid (catalog no. 191504) (MP Biomedicals, Solon, OH), and $\beta$-NAD (catalog no. N1630; SigmaAldrich) were dissolved in water at $0.1 \mathrm{M}$ concentration. MTX (catalog no. 13960; Cayman Chemical Company) was dissolved in 100\% DMSO (catalog no. BP231-100; Thermo Fisher Scientific) at $0.1 \mathrm{M}$ concentration. Olaparib (catalog no. S1060; Selleck Chemicals, Houston, TX) was dissolved in $100 \%$ DMSO at $10 \mathrm{mg} / \mathrm{ml}$ concentration. FK-866 (catalog no. 4808; Tocris Bioscience, Bristol, UK) was dissolved in water at a concentration of $5 \mathrm{mM}$. External reference standards for ZMP (catalog no. A611705; Toronto Research Chemicals, Toronto, ON, Canada) and IMP (catalog no. 226260250; Acros Organics) were dissolved in $100 \%$ acetonitrile.

Statistical Analysis. Data are shown as the mean \pm S.D. in all figures. Associations between continuous variables were assessed by simple linear regression with log transformation of non-normally distributed variables. Data were compared by unpaired $t$ test analysis and significance was determined at $P<0.05$.

\section{Results}

Effect of NAMPT Inhibition on MTX Activity. Previous work by our group demonstrated that siRNA-based silencing of NAMPT and pharmacological inhibition of NAMPT with FK-866 both result in a significant and similar increase in sensitivity to the growth inhibitory effects of MTX in the A549 human lung carcinoma cell line (Funk et al., 2016). To demonstrate the relevance of inhibition of NAMPT on MTX activity in primary human tissues, we employed primary human fibroblasts and PBMCs to evaluate the effect of NAMPT inhibition on MTX response. Utilizing the siRNA-based silencing approach in the 


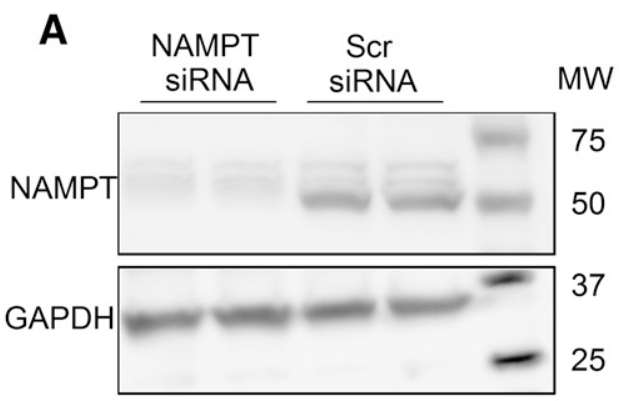

B

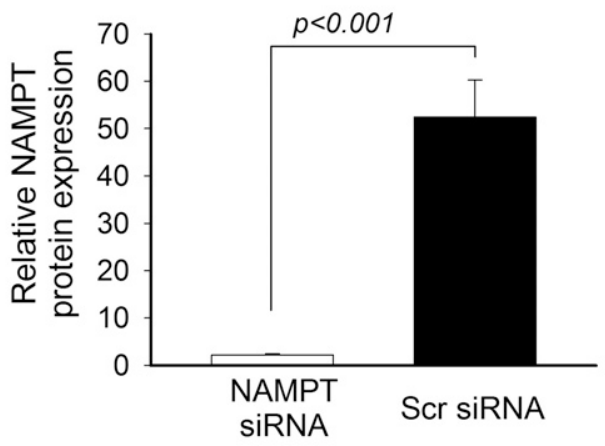

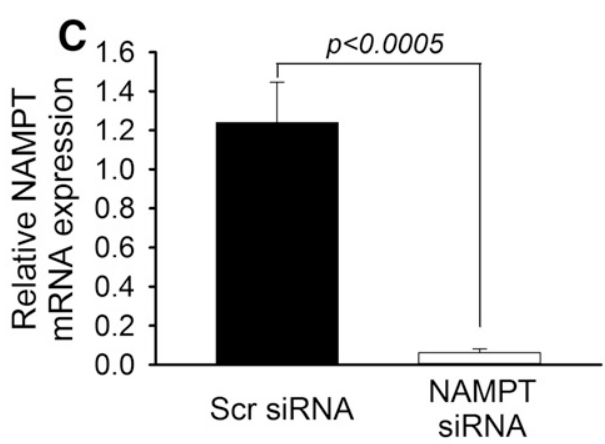

D

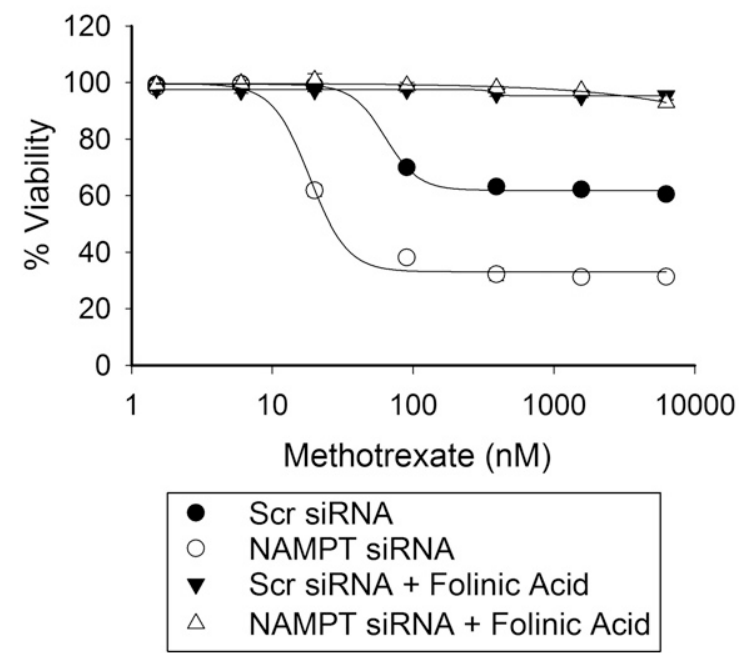

Fig. 2. MTX activity in NAMPT-deficient cells is folate dependent. A549 cells were transfected with either control scrambled siRNA or with siRNA directed toward NAMPT. Twenty-four hours after transfection, cells were treated with different concentrations of MTX alone or in combination with $10 \mu \mathrm{M}$ folinic acid for 96 hours. (A-C) Knockdown of NAMPT by siRNA was confirmed by Western blot analysis (A), quantified by densitometry analysis (B), and verified by real-time-PCR (C). Cell viability was measured by fluorescence spectroscopy using the resazurin reduction assay. (D) The effect of MTX toxicity alone or in combination with $10 \mu \mathrm{M}$ folinic acid was determined as percent viability based on untreated control cells. Experiments were conducted in triplicate and the resulting mean \pm S.D. for each experiment is presented. Concentrations of MTX required for half-maximal inhibition of $50 \%$ inhibition of cell viability were determined and compared by $t$ test analysis. GAPDH, glyceraldehyde-3-phosphate dehydrogenase; MW, molecular weight; Scr, scrambled.

AG07095 human fibroblast cell line, we found that silencing of NAMPT resulted in a significant increase in sensitivity to the growth inhibitory effects of MTX (Fig. 1A). Notably, fibroblasts treated with control siRNA failed to demonstrate any measureable level of growth inhibition after a 96-hour treatment with MTX at concentrations up to $10 \mu \mathrm{M}$. In contrast, fibroblasts treated with NAMPT siRNA demonstrated approximately $25 \%$ growth inhibition with half-maximal response at a mean MTX concentration of $49.1 \pm 14.7 \mathrm{nM}$. Similarly, we found that treatment of isolated PBMCs with 10 nM MTX or 0.1 nM FK-866 individually had no impact on their response to stimulation after treatment with phytohemagglutinin (Fig. 1B). However, treatment with MTX in combination with FK-866 resulted in a synergistic inhibition of lymphocyte proliferation and further supports a synergistic relationship whereby variations in NAMPT activity impact response to MTX in a diversity of tissues.

MTX Activity in NAMPT-Deficient Cells Is Folate Dependent. The antiproliferative activity of MTX is primarily mediated through the competitive inhibition of DHFR resulting in depletion of the intracellular pool of bioactive folates; however, folate-independent mechanisms of action have been proposed (Dolezalová et al., 2005; Funk et al., 2013;
Sramek et al., 2017). The antifolate effects of MTX are reversible through supplementation with the reduced and methylated form of folate, folinic acid, also referred to as 5-formyl tetrahydrofolate (Shea et al., 2014; Koh et al., 2016). Therefore, initial studies were performed to confirm that the antiproliferative activity of MTX in NAMPT-deficient cells was mediated through the antifolate activity of MTX. Consistent with previous results (Funk et al., 2016), siRNA-based silencing of NAMPT resulted in reduced expression of NAMPT in A549 cells, as demonstrated by Western blot analysis (Fig. 2A). By densitometry, the NAMPT band was normalized to glyceraldehyde-3-phosphate dehydrogenase and demonstrated a greater than $95 \%$ reduction in cellular NAMPT protein (Fig. 2B) and was consistent with depletion of NAMPT mRNA as measured by real-time-PCR (Fig. 2C). Similar to our previous finding, silencing of NAMPT resulted in a greater than 3-fold reduction in the concentration of MTX, resulting in half-maximal inhibition $\left(\mathrm{IC}_{50}\right)$ of cell growth at 96 hours (mean $66.9 \pm 12$ vs. $18.5 \pm 0.3 \mathrm{nM}, P<0.005$ ) (Fig. $2 \mathrm{D}$ ). Supplementation of growth media with $10 \mu \mathrm{M}$ folinic acid completely rescued both control cells and NAMPT-deficient cells from the antiproliferative activity of MTX (Fig. 2D). 

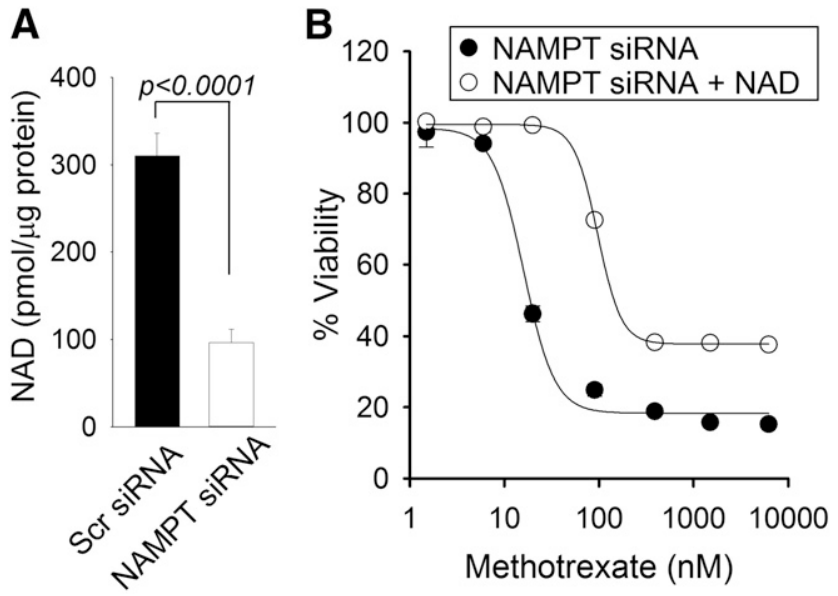

Fig. 3. NAD supplementation reverses enhanced MTX sensitivity in NAMPT-deficient cells. (A) A549 cells were transfected with either control scrambled siRNA or with siRNA directed toward NAMPT and cellular NAD levels were measured 96 hours after transfection. (B) Cellular viability was measured in NAMPT-deficient cells and treated with various concentrations of MTX with or without supplementation of $200 \mu \mathrm{M}$ NAD. Experiments were conducted in triplicate and the resulting mean \pm S.D. for each experiment is presented. NAD levels were compared by $t$ test analysis. Concentrations of MTX required for half-maximal inhibition of $50 \%$ inhibition of cell viability were determined and compared by $t$ test analysis. Scr, scrambled.

NAD Supplementation Reverses MTX Sensitivity in NAMPT-Deficient Cells. Consistent with our previously reported findings, siRNA-based silencing of NAMPT resulted in a marked reduction (approximately 68\%) in cellular NAD levels (Fig. 3A) (Funk et al., 2016). Therefore, to verify the role of NAD depletion as the basis for enhanced MTX sensitivity in NAMPT knockdown cells, we measured the effect of NAD supplementation on MTX response in NAMPT-deficient cells (Fig. 3B). The addition of $200 \mu \mathrm{M}$ NAD in cell culture media caused a 5 -fold increase in the $\mathrm{IC}_{50}$ values for growth inhibition of MTX in NAMPT-deficient cells (mean $17.92 \pm 2.05$ vs. $95.6 \pm 4.50 \mathrm{nM}, P=0.00001)$ that were similar to those observed previously in control cells with normal NAMPT levels. In contrast, supplementation of NAD in control cells with normal NAMPT and NAD levels resulted in no significant effect on MTX response (data not shown).

NAMPT Deficiency Does Not Disrupt Folate Homeostasis or Increase MTX Uptake. The phosphorylated and reduced form of NAD (NADPH) is necessary for proper folding and stability of DHFR and serves as a cofactor in the reduction of dihydrofolate to tetrahydrofolate (THF) (Ainavarapu et al., 2005; Hsieh et al., 2013). As a result, decreases in cellular NADPH would be expected to result in both the reduced expression of DHFR as well as reduced enzymatic activity. Recognizing that inhibition of NAMPT activity results in the depletion of both cellular NAD and $\mathrm{NADP}(\mathrm{H})$ pools (Hsieh et al., 2013), it is reasonable to hypothesize that depletion of cellular NADPH secondary to reduced NAMPT activity would increase sensitivity to MTX through a reduction in DHFR expression and activity. To determine the effect of reductions in cellular NAMPT levels on the expression of DHFR, both control and NAMPT-deficient cells were evaluated for DHFR expression by Western blot analysis (Fig. 4A). Densitometry analysis confirmed that reductions in NAMPT expression had no effect on the cellular levels of DHFR (Fig. 4B). DHFR activity was assessed by measuring cellular levels of THF and its methylated metabolites 5-methyl-THF and 5,10-methenylTHF (Fig. 4C). Cellular folate levels in NAMPT-deficient cells were not consistent with a reduction in DHFR activity and actually resulted in an increase in cellular folate levels but were not statistically significant. Furthermore, to evaluate whether the increased sensitivity to MTX potentially resulted from an increase in the cellular uptake of MTX in NAMPTdeficient cells, concentrations of MTX and its polyglutamated metabolites were measured in control and NAMPT-deficient cells (Fig. 4D). NAMPT-deficient cells did not take up more MTX; in contrast, they appeared to have lower levels of the drug and its pharmacologically active metabolites compared with the control cells.

MTX Does Not Cause Synergistic Depletion of Cellular NAD in NAMPT-Deficient Cells. Although the increased sensitivity of NAMPT-deficient cells appears to result from a reduction in the enzymatic function of NAMPT causing the cellular depletion of NAD, the biochemical basis for increased MTX activity in NAMPT-deficient cells remains unclear. As discussed in the Introduction, a previous study reported that the antifolate agent pemetrexed promotes the cytotoxicity of a chemical inhibitor of NAMPT through activation of PARP-1 in response to DNA damage by pemetrexed, leading to the synergistic depletion of cellular NAD (Chan et al., 2014). In contrast to this previous work, MTX was not found to cause any measurable activation of PARP-1 as monitored by Western blot analysis for the production of PAR (Fig. 5A). In agreement with these findings, treatment with MTX did not cause any significant DNA damage as measured by DNA gel electrophoresis (Fig. 5B). Finally, although MTX caused a slight but insignificant reduction in cellular NAD levels in control cells, MTX failed to cause any further depletion of NAD levels in the NAMPT-deficient cells (Fig. 5C).

Furthermore, to rule out involvement of PARP-1 activation by MTX as the mechanism of increased response to MTX, the antiproliferative activity of MTX was measured in the presence of the PARP inhibitor olaparib, which was previously demonstrated to rescue pemetrexed toxicity in cells treated with a NAMPT inhibitor (Chan et al., 2014). We did not find any significant change in the growth inhibition of MTX in NAMPT-deficient or control cells in the presence of olaparib (Fig. 6).

Increased MTX Activity Occurs through Synergistic Depletion of Cellular ATP. As an antifolate, MTX is a potent inhibitor of folate-dependent enzymes responsible for de novo purine and pyrimidine biosynthesis (Fairbanks et al., 1999; Budzik et al., 2000; Kremer, 2004). As a result, MTX causes the exposure-dependent depletion of the intracellular nucleotide pool, including the depletion of intracellular ATP (Budzik et al., 2000; Chan et al., 2014). Pharmacological inhibition of NAMPT also ultimately causes the depletion of intracellular ATP, secondary to the depletion of cellular NAD, and has been directly associated with cytotoxicity (Tan et al., 2013; Moore et al., 2015). Therefore, we investigated whether the increased antiproliferative effect of MTX in NAMPTdeficient cells is associated with synergistic depletion of the cellular ATP pool. Cellular ATP levels in control and NAMPTdeficient cells were measured, and interestingly, NAMPTdeficient cells had significantly higher levels of ATP compared 

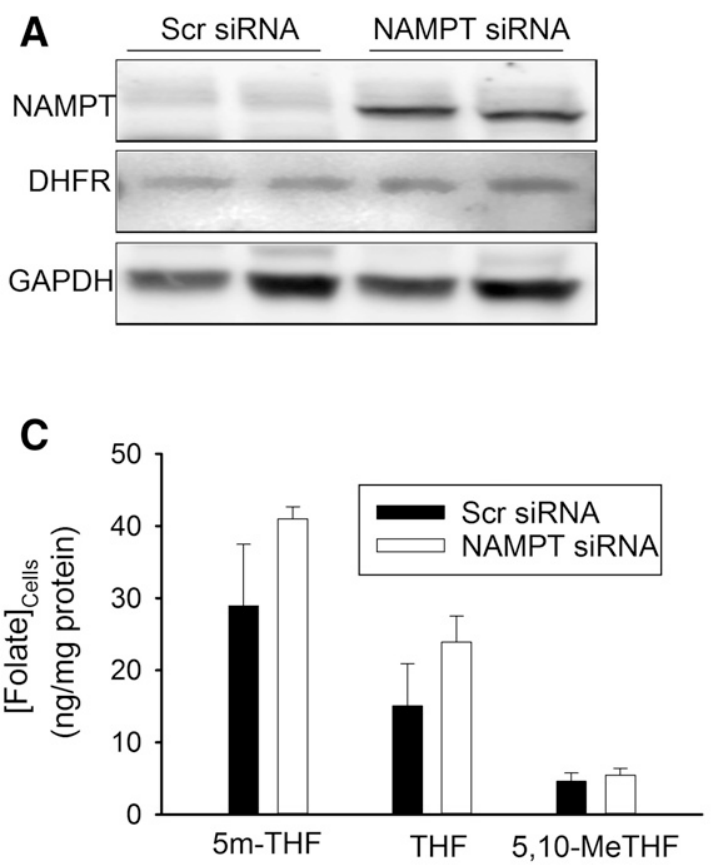
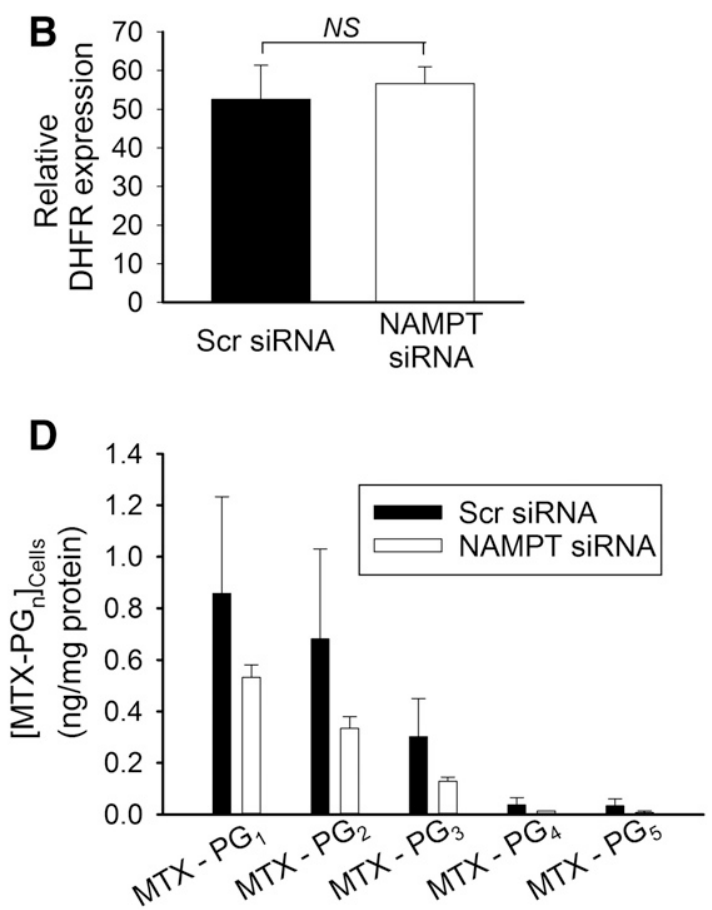

Fig. 4. Inhibition of NAMPT does not affect DHFR expression or intracellular levels of folate or MTX. (A) A549 cells were transfected with either control scrambled siRNA or with siRNA directed toward NAMPT and protein levels of DHFR were monitored 96 hours after transfection. (B) For densitometry analysis, DHFR protein levels were normalized to GAPDH. (C and D) Cellular concentrations of folate (C) and MTX levels (D) were analyzed in control and NAMPT-deficient cells treated for 24 hours with $1000 \mathrm{nM}$ MTX. Experiments were conducted in triplicate and the resulting mean \pm S.D. for each experiment is presented and compared by $t$ test analysis. GAPDH, glyceraldehyde-3-phosphate dehydrogenase; NS, not significant; Scr, scrambled.

with control cells (Fig. 7A). Increased cellular ATP content in response to NAMPT inhibition was previously reported in cells that solely rely on salvage or the nicotinamide pathway for NAD synthesis (Tolstikov et al., 2014). Treatment with MTX over 96 hours resulted in the dose-dependent depletion of cellular ATP in both control and NAMPT-deficient cells (Fig. 7B). However, NAMPT-deficient cells were significantly more sensitive to the ATP-depleting activity of MTX. Similar to the enhanced antiproliferative effects seen with NAMPT deficiency, reduced NAMPT expression resulted in a nearly 4-fold reduction in the concentration of MTX, resulting in halfmaximal depletion of cellular ATP (mean $15.01 \pm 3.63 \mathrm{vs} .3 .93$ $\pm 0.35 \mathrm{nM}, P<0.005$ ).

An analysis of the intermediates of the de novo purine synthesis pathway was undertaken to evaluate whether NAMPT deficiency impacts the activity of aminoimidazole carboxamide ribonucleotide transformylase (AICART), which is one of the primary intracellular targets of MTX in the inhibition of de novo purine biosynthesis (Allegra et al., 1985). To evaluate AICART activity, we measured both the substrate (ZMP) and the product (IMP) of AICART in NAMPT-deficient and control cells (Fig. 8). Interestingly, intracellular levels of both ZMP and IMP were significantly elevated in NAMPTdeficient cells. However, a comparison of the ratio of IMP/ZMP as a static measure of AICART enzymatic activity revealed no significant difference between NAMPT-deficient and control cells.

Supplementation with intermediates of purine and pyrimidine biosynthesis can restore cellular nucleotide pools depleted in response to treatment with MTX (Piper et al., 1983; Chan and Howell, 1990). Therefore, to investigate whether depletion of nucleotide precursors by MTX is responsible for the increase in pharmacological activity observed in response to NAMPT inhibition, we evaluated cell viability, NAD levels, and ATP levels in control and NAMPT-deficient cells treated with MTX, with and without supplemental HYP and THY (Fig. 9). Under the conditions tested, supplementation with HYP and THY completely rescued the antiproliferative effects of MTX in both control and NAMPT knockdown cells (Fig. 9A). The observed increase in cell viability corresponded to a marked increase in cellular ATP levels in both the control and NAMPT knockdown cells (Fig. 9B). However, the reversal in the antiproliferative activity of MTX with HYP-THY supplementation had no effect on NAD levels in the NAMPT-deficient cells (Fig. 9C).

\section{Discussion}

Based on the results of this study, we conclude that reductions in the enzymatic activity of NAMPT increase the sensitivity of cells to the inhibition of nucleotide biosynthesis by MTX and potentiate the MTX-mediated depletion of cellular ATP. Together, these findings illustrate a novel mechanism through which disruption of cellular NAD metabolism, through reduction in the enzymatic activity of NAMPT, enhances the pharmacological activity of the antifolate therapeutic MTX. These findings suggest that variation in NAMPT expression and NAD homeostasis, reflecting both potential genetic and environmental factors, may impact the sensitivity of patients to the pharmacological effects of MTX, and is supported by our previous clinical finding that patients with reduced circulating NAMPT levels are more likely to respond to MTX (Funk et al., 2016). 

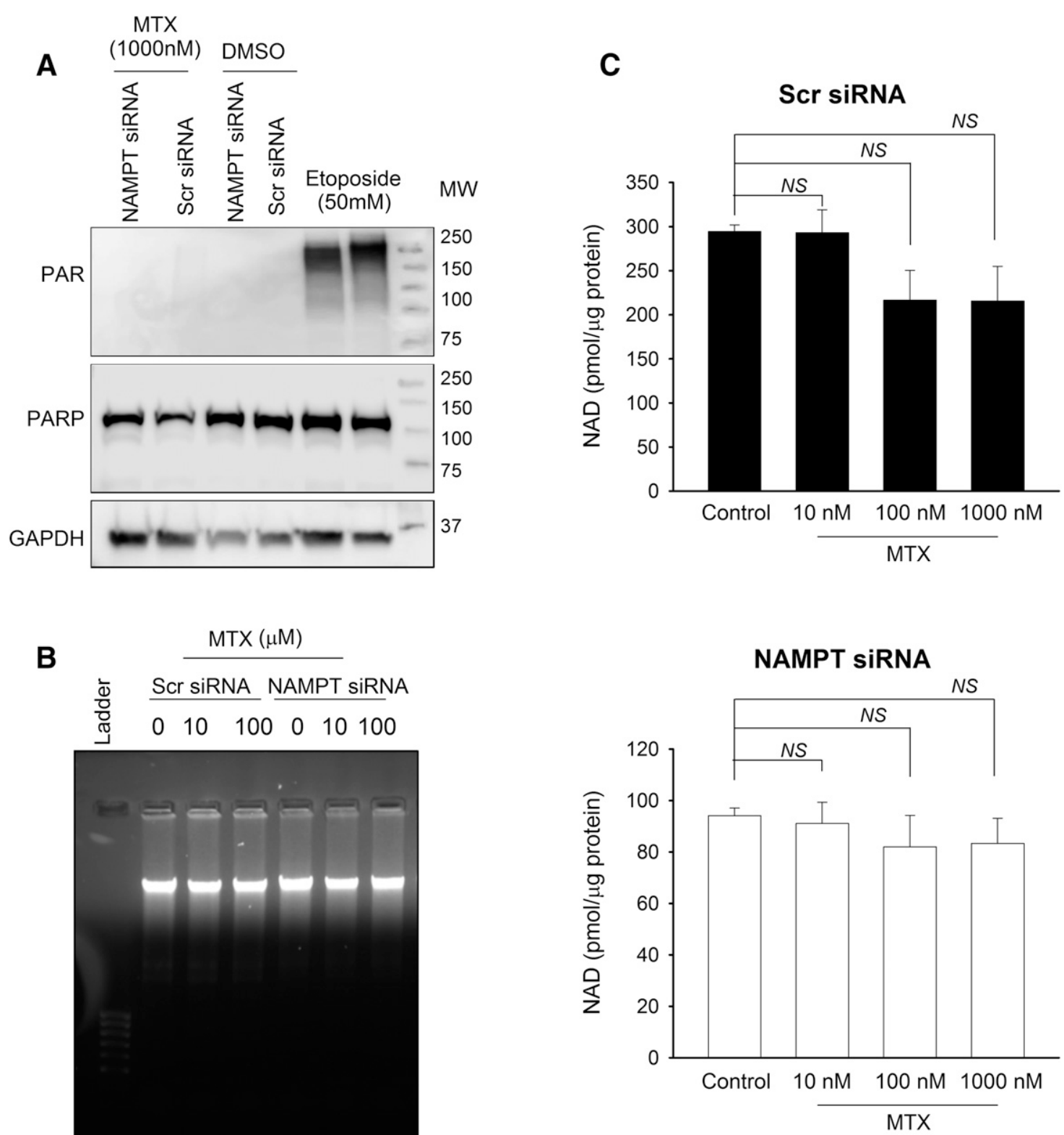

Fig. 5. MTX does not cause synergistic depletion of cellular NAD in NAMPT-deficient cells. (A) A549 cells were transfected with either control scrambled siRNA or with siRNA directed toward NAMPT; 24 hours after transfection, cells were treated with $1000 \mathrm{nM}$ MTX for 96 hours or 50 mM etoposide for 24 hours to determine activation of PARP by measuring PAR formation by Western blot analysis. (B) Control and NAMPT-deficient cells were treated with different concentrations of MTX for 96 hours and total DNA was separated on $1 \%$ agarose gel to evaluate DNA damage. (C) Total NAD levels were monitored in control and NAMPT-deficient cells treated with different concentrations of MTX for 96 hours. Experiments were conducted in triplicate and the resulting mean \pm S.D. for each experiment is presented and compared by $t$ test analysis. GAPDH, glyceraldehyde-3-phosphate dehydrogenase; MW, molecular weight; NS, not significant; PAR, poly(ADP-ribose); Scr, scrambled.

Our initial experiments sought to demonstrate the interaction of NAMPT and MTX in primary tissues and to determine whether the enhanced sensitivity to MTX observed with NAMPT-deficient cells was mediated through the antifolate activity of MTX. We found that inhibition of NAMPT activity potentiated the growth inhibitory effects of MTX in both primary human fibroblasts and isolated lymphocytes (Fig. 1 ), and we therefore demonstrated that this interaction is broadly applicable to both transformed and primary tissues. Although MTX is widely believed to mediate its pharmacological activity through inhibition of folate-dependent biochemical pathways, either through the depletion of the intracellular pool of bioactive folates or through the direct inhibition of the various folate-dependent enzymes, some investigators have suggested folate-independent pharmacological effects of MTX mediated through inhibition of Janus kinase/signal transducers and activators of transcription signaling, induction of oxidative stress, and mitochondrial toxicity (Phillips et al., 2003; Thomas et al., 2015; Zimmerman et al., 2017; Al Maruf et al., 2018). Therefore, we evaluated whether supplementation with folinic acid would reverse the increased antiproliferative effects of MTX in NAMPT-deficient cells (Fig. 2). Our finding that folinic acid supplementation completely rescues NAMPT-deficient cells from the antiproliferative effects of 

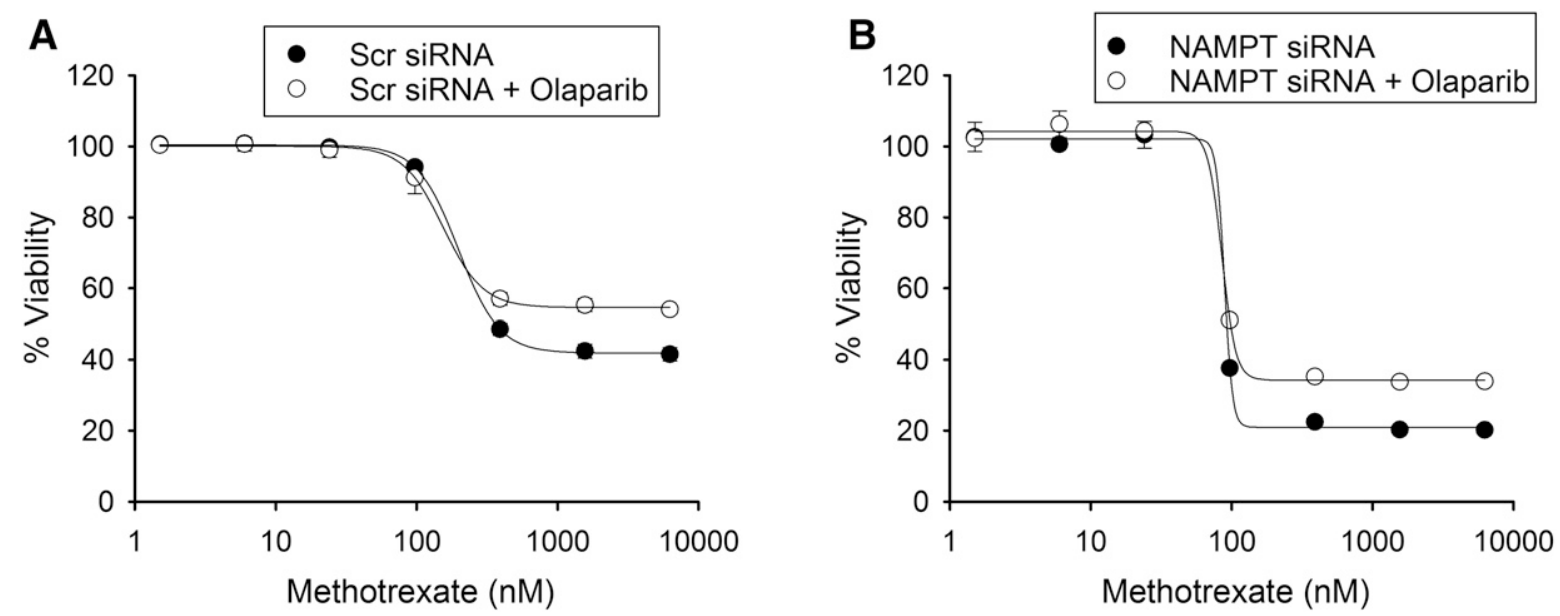

Fig. 6. Increased MTX activity does not occur through activation of PARP. A549 cells transfected with either control scrambled siRNA or with siRNA directed toward NAMPT were treated with different concentrations of MTX with or without $10 \mu \mathrm{M}$ olaparib. (A and B) The effect of inhibition of PARP-1 by olaparib on MTX toxicity in control (A) and NAMPT-deficient (B) cells was determined by normalizing cell growth inhibition with MTX against control cells. Experiments were conducted in triplicate and the resulting mean \pm S.D. for each experiment is presented. Concentrations of MTX required for half-maximal inhibition of 50\% inhibition of cell viability were determined and compared by $t$ test analysis. Scr, scrambled.

MTX suggests that the enhanced sensitivity of cells with reduced NAMPT activity is a result of increased sensitivity to the antifolate effects of MTX and is not mediated through a secondary folate-independent pharmacological mechanism. Similarly, recognizing that much debate continues on the biologic function of NAMPT beyond its role as the rate-limiting enzyme in NAD biosynthesis (Revollo et al., 2004; Rongvaux et al., 2008; Moore et al., 2015; Chen et al., 2016), we sought to determine whether the depletion of cellular NAD in NAMPTdeficient cells was responsible for the observed increase in sensitivity to the antiproliferative activity of MTX. Therefore, the effect of NAD supplementation on the antiproliferative effect of MTX was measured in NAMPT-deficient cells (Fig. 3). Our finding that NAD supplementation of NAMPT-deficient cells reversed the observed increase in sensitivity to the antiproliferative effects of MTX established that reductions in the enzymatic activity of NAMPT were responsible for the increased sensitivity toward MTX and that the effect was not a manifestation of one of the multitude or purported nonenzymatic functions of NAMPT. Together, these data established that reduced cellular concentrations of NAD, resulting from deficiency in the enzymatic activity of NAMPT, increase the sensitivity of cells to the antifolate-mediated antiproliferative effects of MTX. Furthermore, these data support a mechanistic interaction through which regulation of cellular NAD can impact the sensitivity of cells to the pharmacological activity of antifolate therapeutics.

Recognizing that a multitude of mechanisms exist through which cells can display enhanced or diminished sensitivity to MTX (Cutolo et al., 2001; Kremer, 2004), we sought to understand the mechanism through which reductions in cellular NAD increase the sensitivity of cells to the antifolate effects of MTX. Among the most obvious potential mechanisms would be reductions in DHFR expression or activity, which were previously demonstrated as an important factor impacting the sensitivity of cells to the pharmacological activity of MTX (Cutolo et al., 2001; Hsieh et al., 2013). Recognizing that NADPH is depleted through inhibition of NAMPT and that NADPH is required for the enzymatic activity and stabilization of DHFR, we sought to evaluate the effect of reduced NAMPT activity on DHFR (Hsieh et al., 2013). However, we failed to find a change in DHFR protein levels in NAMPT-deficient cells (Fig. 4). We also found no significant change in the intracellular concentrations of the major bioactive forms of folate in NAMPT-deficient cells (Fig. 4). Therefore, neither the expression nor the activity of DHFR played a role in the observed increase in sensitivity to MTX in NAMPT-deficient cells.

From a pharmacological standpoint, differences in the cellular uptake and polyglutamation of MTX are the other major mechanism that has been commonly associated with differences in sensitivity to MTX (Tian and Cronstein, 2007;
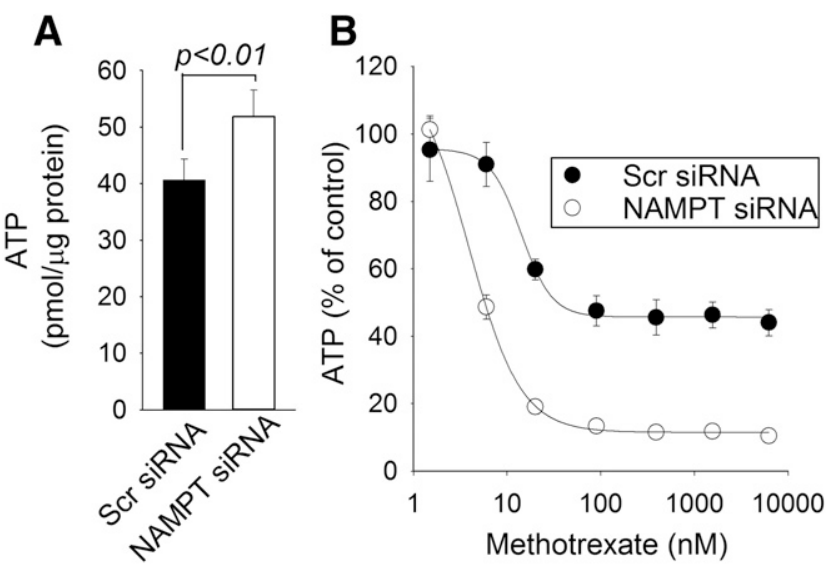

Fig. 7. NAMPT inhibition results in the synergistic depletion of cellular ATP by MTX. (A and B) A549 cells transfected with either control scrambled siRNA or with siRNA directed toward NAMPT were treated with different concentrations of MTX and cellular ATP levels were measured 96 hours after transfection (A) and in response to treatment with different concentrations of MTX over 96 hours (B). The effect of MTX on cellular ATP was determined by normalizing cellular ATP levels to the untreated control cells. Experiments were conducted in triplicate and the resulting mean \pm S.D. for each experiment is presented and compared by $t$ test analysis. Scr, scrambled. 

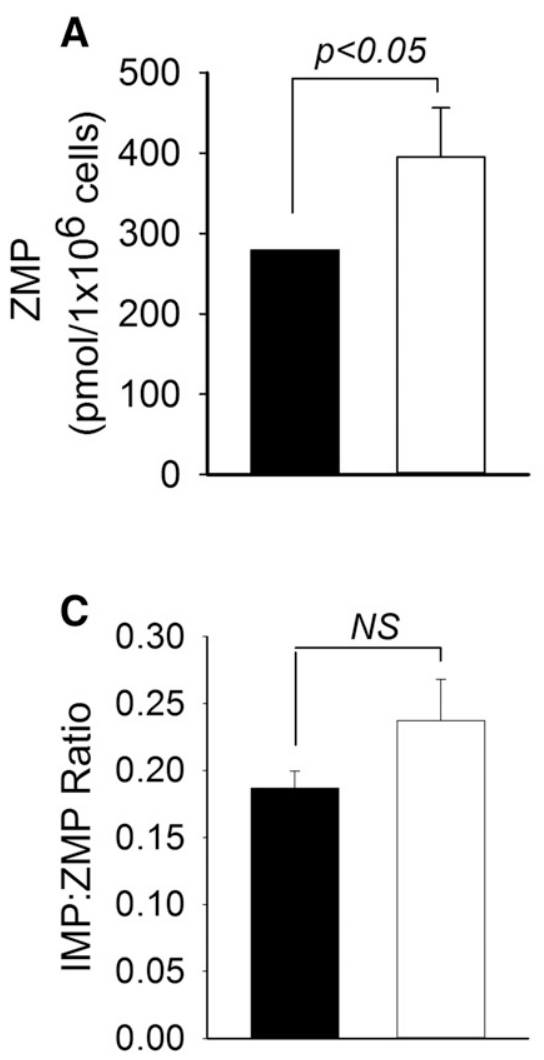

Funk et al., 2013, 2014). As a result, we measured the cellular levels of MTX and its polyglutamated metabolites in NAMPTdeficient cells and found no significant difference compared with control cells (Fig. 4). Therefore, our data suggest that the

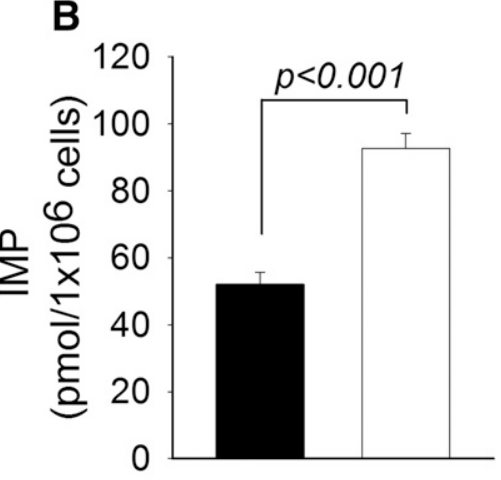

Fig. 8. NAMPT inhibition does not affect AICART activity but is associated with an increase in intracellular intermediates of de novo purine biosynthesis. A549 cells were transfected with either control scrambled siRNA or with NAMPT-specific siRNA. (A-C) After 72 hours under normal culture conditions, cells were subsequently harvested and intracellular concentrations of ZMP (A) and IMP (B) and the ratio of IMP/ ZMP $(\mathrm{C})$ were determined. Experiments were conducted in triplicate and the resulting mean \pm S.D. for each experiment is presented and compared by $t$ test analysis. NS, not significant; Scr, scrambled.

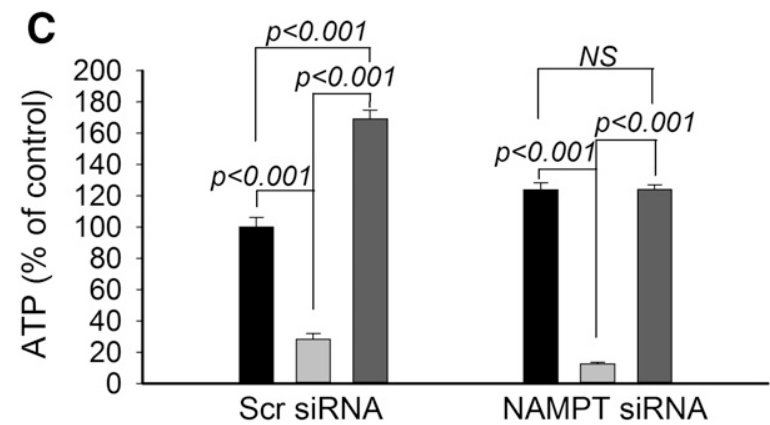

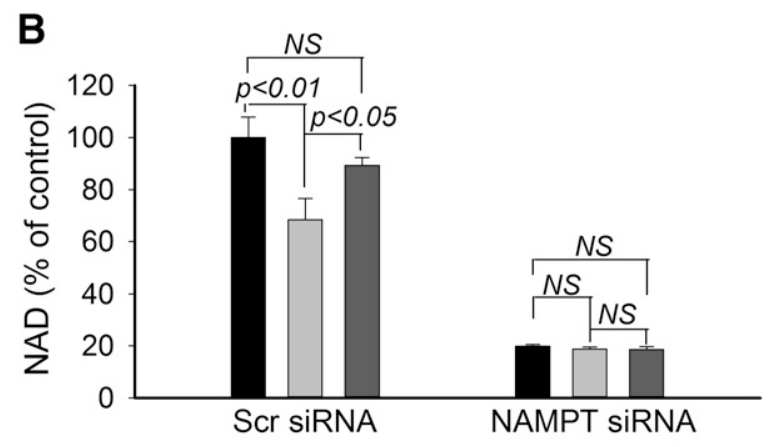

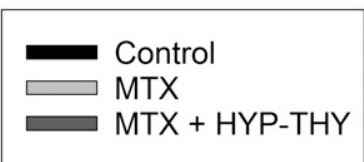

Fig. 9. Supplementation of nucleotide biosynthesis rescues cell viability and ATP levels, but not NAD levels. (A-C) A549 cells transfected with either control scrambled siRNA or with siRNA directed toward NAMPT were treated with $1000 \mathrm{nM}$ MTX alone or together with a combination of $100 \mu \mathrm{M}$ hypoxanthine and $100 \mu \mathrm{M}$ thymidine for 96 hours and measured for cellular viability (A), cellular NAD levels (B), and cellular ATP levels (C). Experiments were conducted in triplicate and the resulting mean \pm S.D. for each experiment is presented and compared by $t$ test analysis. NS, not significant; Scr, scrambled. 
cells must occur through a mechanism that is downstream from regulation of MTX disposition and folate homeostasis.

Although depletion of cellular NAD is not a commonly reported effect of MTX therapy, a previous report that the antifolate pemetrexed potentiated the cytotoxic effects of GMX1777 through the synergistic depletion of cellular NAD led us to evaluate the effect of MTX on NAD levels in our NAMPT-deficient cells (Chan et al., 2014). In our evaluations, we found that MTX had no effect on cellular NAD in NAMPTdeficient cells and had only a modest effect in control cells (Figs. 5 and 9). To further confirm these findings, we evaluated NAMPT-deficient cells for evidence of direct DNA damage, the activation of PARP-1, and the effect of PARP-1 inhibition on the antiproliferative activity of MTX. We found no evidence of MTX-mediated DNA damage, PARP-1 activation, or reversal of MTX activity by inhibition of PARP-1 (Figs. 5 and 6). In addition, there was no effect on the antiproliferative activity of MTX when control cells were supplemented with NAD, which further suggests that MTX does not mediate its antiproliferative activity through depletion of NAD under normal conditions. These findings suggest that the increased antiproliferative activity of MTX in NAMPT-deficient cells does not result from the synergistic depletion of NAD through activation of PARP-1, as was previously demonstrated for the interaction between pemetrexed and GMX1777 (Chan et al., 2014).

The antiproliferative activity of MTX is primarily associated with the inhibition of de novo purine and pyrimidine biosynthesis mediated through the inhibition of phosphoribosylglycineamide formyltransferase and AICART in the purine biosynthesis pathway and thymidylate synthase in the pyrimidine biosynthesis pathway (Allegra et al., 1985; Baram et al., 1988; Sant et al., 1992). Inhibition of nucleotide biosynthesis by MTX was shown to result in the depletion of cellular ATP at physiologic relevant MTX concentrations and was associated with its antiproliferative activity (Smoleńska et al., 1999; Chan et al., 2014). Therefore, we sought to evaluate cellular levels of ATP in response to MTX treatment in NAMPT-deficient cells and found these cells to be approximately 4-fold more sensitive to the ATP-depleting effects of MTX compared with control cells (Fig. 7). Interestingly, the fold variation in ATP-depleting activity and antiproliferative activity was similar between NAMPT-deficient and control cells and suggests a relationship between the increased sensitivity of NAMPT-deficient cells to the depletion of ATP by MTX and the antiproliferative activity of MTX. To investigate the possibility that NAMPT-deficient cells had altered AICART activity, we measured intracellular concentrations of ZMP and IMP and calculated the IMP/ZMP ratio (Fig. 8). We found no difference in AICART activity in NAMPT-deficient and control cells. However, we found that NAMPT-deficient cells had higher intracellular concentrations of both ZMP and IMP, which indicated an increase in de novo purine biosynthesis in NAMPT-deficient cells that could foreseeably make them more vulnerable to the growth inhibitory effects of MTX mediated through the inhibition of purine biosynthesis. Furthermore, to verify the role of inhibition of nucleotide biosynthesis as the mechanism for the increased antiproliferative activity of MTX, we supplemented NAMPT-deficient cells with intermediates of the nucleotide salvage pathway (Fig. 9). Supplementation of HYP-THY completely prevented the depletion of ATP in NAMPT-deficient cells and was associated with inhibition of the antiproliferative effects of MTX. Furthermore, cellular NAD levels were not affected by supplementation with HYP-THY. Together, these results demonstrate that NAMPT-deficient cells appear to have an increase in demand for de novo purine biosynthesis and are therefore more sensitive to the inhibition of nucleotide biosynthesis by MTX, resulting in enhanced depletion of the intracellular ATP pool.

In conclusion, our findings establish a new functional link between antifolate pharmacology and regulation of cellular NAD by NAMPT and suggest the potential importance of variation in NAMPT activity in the observed variation in clinical response to MTX. An understanding of the regulation of intracellular NAD homeostasis may represent an important factor impacting the pharmacological activity of MTX. Further study is needed to evaluate how this information can be potentially used to improve response to MTX therapy in the treatment of autoimmune arthritis.

\section{Authorship Contributions}

Participated in research design: Singh, Leeder, Becker, Funk. Conducted experiments: Singh, Funk.

Contributed new reagents or analytic tools: van Haandel, Heruth, Ye, Funk.

Performed data analysis: Singh, Funk.

Wrote or contributed to the writing of the manuscript: Singh, Funk.

\section{References}

Ainavarapu SR, Li L, Badilla CL, and Fernandez JM (2005) Ligand binding modulates the mechanical stability of dihydrofolate reductase. Biophys $J$ 89:3337-3344 Al Maruf A, O'Brien PJ, Naserzadeh P, Fathian R, Salimi A, and Pourahmad J (2018) Methotrexate induced mitochondrial injury and cytochrome $\mathrm{c}$ release in rat liver hepatocytes. Drug Chem Toxicol 41:51-61.

Allegra CJ, Drake JC, Jolivet J, and Chabner BA (1985) Inhibition of phosphoribosylaminoimidazolecarboxamide transformylase by methotrexate and dihydrofolic acid polyglutamates. Proc Natl Acad Sci USA 82:4881-4885.

Baram J, Chabner BA, Drake JC, Fitzhugh AL, Sholar PW, and Allegra CJ (1988) Identification and biochemical properties of 10 -formyldihydrofolate, a novel folate found in methotrexate-treated cells. J Biol Chem 263:7105-7111.

Budzik GP, Colletti LM, and Faltynek CR (2000) Effects of methotrexate on nucleotide pools in normal human T cells and the CEM T cell line. Life Sci 66: 2297-2307.

Bulatović M, Heijstek MW, Verkaaik M, van Dijkhuizen EH, Armbrust W, Hoppenreijs EP, Kamphuis S, Kuis W, Egberts TC, Sinnema G, et al. (2011) High prevalence of methotrexate intolerance in juvenile idiopathic arthritis: development and validation of a methotrexate intolerance severity score. Arthritis Rheum 63:2007-2013.

Busso N, Karababa M, Nobile M, Rolaz A, Van Gool F, Galli M, Leo O, So A, and De Smedt T (2008) Pharmacological inhibition of nicotinamide phosphoribosyltransferase/visfatin enzymatic activity identifies a new inflammatory pathway linked to NAD. PLoS One 3:e2267.

Chan M, Gravel M, Bramoullé A, Bridon G, Avizonis D, Shore GC, and Roulston A (2014) Synergy between the NAMPT inhibitor GMX1777(8) and pemetrexed in non-small cell lung cancer cells is mediated by PARP activation and enhanced NAD consumption. Cancer Res 74:5948-5954.

Chan TC and Howell SB (1990) Role of hypoxanthine and thymidine in determining methotrexate plus dipyridamole cytotoxicity. Eur J Cancer 26:907-911.

Chavan H, Li F, Tessman R, Mickey K, Dorko K, Schmitt T, Kumer S, Gunewardena S, Gaikwad N, and Krishnamurthy P (2015) Functional coupling of ATP-binding cassette transporter Abcb6 to cytochrome P450 expression and activity in liver. $J$ Biol Chem 290:7871-7886.

Chen H, Wang S, Zhang H, Nice EC, and Huang C (2016) Nicotinamide phosphoribosyltransferase (Nampt) in carcinogenesis: new clinical opportunities. Expert Rev Anticancer Ther 16:827-838.

Cutolo M, Sulli A, Pizzorni C, Seriolo B, and Straub RH (2001) Anti-inflammatory mechanisms of methotrexate in rheumatoid arthritis. Ann Rheum Dis 60:729-735.

Dolezalová P, Krijt J, Chládek J, Nemcová D, and Hoza J (2005) Adenosine and methotrexate polyglutamate concentrations in patients with juvenile arthritis. Rheumatology (Oxford) 44:74-79.

Evans L, Williams AS, Hayes AJ, Jones SA, and Nowell M (2011) Suppression of leukocyte infiltration and cartilage degradation by selective inhibition of pre-B cell colony-enhancing factor/visfatin/nicotinamide phosphoribosyltransferase: Apo866mediated therapy in human fibroblasts and murine collagen-induced arthritis. Arthritis Rheum 63:1866-1877.

Fairbanks LD, Rückemann K, Qiu Y, Hawrylowicz CM, Richards DF, Swaminathan R, Kirschbaum B, and Simmonds HA (1999) Methotrexate inhibits the first committed step of purine biosynthesis in mitogen-stimulated human T-lymphocytes: a metabolic basis for efficacy in rheumatoid arthritis? Biochem $J$ 342:143-152. 
Friebe D, Neef M, Kratzsch J, Erbs S, Dittrich K, Garten A, Petzold-Quinque S, Blüher S, Reinehr T, Stumvoll M, et al. (2011) Leucocytes are a major source of circulating nicotinamide phosphoribosyltransferase (NAMPT)/pre-B cell colony $(\mathrm{PBEF}) /$ visfatin linking obesity and inflammation in humans. Diabetologia 54: $1200-1211$.

Funk RS, Singh R, Pramann L, Gigliotti N, Islam S, Heruth DP, Ye SQ, Chan MA Leeder JS, and Becker ML (2016) Nicotinamide phosphoribosyltransferase attenuates methotrexate response in juvenile idiopathic arthritis and in vitro. Clin Transl Sci 9:149-157.

Funk RS, van Haandel L, Becker ML, and Leeder JS (2013) Low-dose methotrexate results in the selective accumulation of aminoimidazole carboxamide ribotide in an erythroblastoid cell line. J Pharmacol Exp Ther 347:154-163.

Funk RS, van Haandel L, Leeder JS, and Becker ML (2014) Folate depletion and increased glutamation in juvenile idiopathic arthritis patients treated with methotrexate. Arthritis Rheumatol 66:3476-3485.

Hsieh YC, Tedeschi P, Adebisi Lawal R, Banerjee D, Scotto K, Kerrigan JE, Lee KC, Johnson-Farley N, Bertino JR, and Abali EE (2013) Enhanced degradation of dihydrofolate reductase through inhibition of NAD kinase by nicotinamide analogs. Mol Pharmacol 83:339-353.

Koh KT, Teh CL, Cheah CK, Ling GR, Yong MC, Hong HC, and Gun SC (2016) Realworld experiences of folic acid supplementation (5 versus $30 \mathrm{mg} /$ week) with methotrexate in rheumatoid arthritis patients: a comparison study. Reumatismo 68:90-96.

Kremer JM (2004) Toward a better understanding of methotrexate. Arthritis Rheum 50:1370-1382.

Liakos CI, Sanidas EA, Perrea DN, Grassos CA, Chantziara V, Viniou NA, Barbetseas JD, and Papadopoulos DP (2016) Apelin and visfatin plasma levels in healthy individuals with high normal blood pressure. Am J Hypertens 29:549-552.

Mirfeizi Z, Noubakht Z, Rezaie AE, Jokar MH, and Sarabi ZS (2014) Plasma levels of leptin and visfatin in rheumatoid arthritis patients; is there any relationship with joint damage? Iran J Basic Med Sci 17:662-666.

Moore Z, Chakrabarti G, Luo X, Ali A, Hu Z, Fattah FJ, Vemireddy R, DeBerardinis RJ, Brekken RA, and Boothman DA (2015) NAMPT inhibition sensitizes pancreatic adenocarcinoma cells to tumor-selective, PAR-independent metabolic catastrophe and cell death induced by $\beta$-lapachone. Cell Death Dis 6:e1599.

Moschen AR, Gerner RR, and Tilg H (2010) Pre-B cell colony enhancing factor/ NAMPT/visfatin in inflammation and obesity-related disorders. Curr Pharm Des 16:1913-1920.

Otero M, Lago R, Gomez R, Lago F, Dieguez C, Gómez-Reino JJ, and Gualillo O (2006) Changes in plasma levels of fat-derived hormones adiponectin, leptin, resistin and visfatin in patients with rheumatoid arthritis. Ann Rheum Dis 65 1198-1201.

Phillips DC, Woollard KJ, and Griffiths HR (2003) The anti-inflammatory actions of methotrexate are critically dependent upon the production of reactive oxygen species. Br J Pharmacol 138:501-511.

Piper AA, Nott SE, Mackinnon WB, and Tattersall MH (1983) Critical modulation by thymidine and hypoxanthine of sequential methotrexate-5-fluorouracil synergism in murine L1210 cells. Cancer Res 43:5101-5105.

Présumey J, Courties G, Louis-Plence P, Escriou V, Scherman D, Pers YM, Yssel H, Pène J, Kyburz D, Gay S, et al. (2013) Nicotinamide phosphoribosyltransferase/ visfatin expression by inflammatory monocytes mediates arthritis pathogenesis. Ann Rheum Dis 72:1717-1724.
Ravelli A, Gerloni V, Corona F, Falcini F, Lepore L, De Sanctis R, Zulian F, Buoncompagni A, Sardella ML, Strano CG, et al.; Italian Pediatric Rheumatology Study Group (1998) Oral versus intramuscular methotrexate in juvenile chronic arthritis. Clin Exp Rheumatol 16:181-183.

Revollo JR, Grimm AA, and Imai S (2004) The NAD biosynthesis pathway mediated by nicotinamide phosphoribosyltransferase regulates Sir2 activity in mammalian cells. J Biol Chem 279:50754-50763.

Rongvaux A, Galli M, Denanglaire S, Van Gool F, Drèze PL, Szpirer C, Bureau F, Andris F, and Leo O (2008) Nicotinamide phosphoribosyl transferase/pre-B cell colony-enhancing factor/visfatin is required for lymphocyte development and cellular resistance to genotoxic stress. J Immunol 181:4685-4695.

Samal B, Sun Y, Stearns G, Xie C, Suggs S, and McNiece I (1994) Cloning and characterization of the cDNA encoding a novel human pre-B-cell colony-enhancing factor. Mol Cell Biol 14:1431-1437.

Sant ME, Lyons SD, Phillips L, and Christopherson RI (1992) Antifolates induce inhibition of amido phosphoribosyltransferase in leukemia cells. J Biol Chem 267 11038-11045.

Shea B, Swinden MV, Ghogomu ET, Ortiz Z, Katchamart W, Rader T, Bombardier C, Wells GA, and Tugwell P (2014) Folic acid and folinic acid for reducing side effects in patients receiving methotrexate for rheumatoid arthritis. $J$ Rheumatol 41: $1049-1060$

Smoleńska Z, Kaznowska Z, Zarówny D, Simmonds HA, and Smoleński RT (1999) Effect of methotrexate on blood purine and pyrimidine levels in patients with rheumatoid arthritis. Rheumatology (Oxford) 38:997-1002.

Sramek M, Neradil J, and Veselska R (2017) Much more than you expected: the nonDHFR-mediated effects of methotrexate. Biochim Biophys Acta 1861:499-503.

Tan B, Young DA, Lu ZH, Wang T, Meier TI, Shepard RL, Roth K, Zhai Y, Huss K, Kuo MS, et al. (2013) Pharmacological inhibition of nicotinamide phosphoribosyltransferase (NAMPT), an enzyme essential for NAD+ biosynthesis, in human cancer cells: metabolic basis and potential clinical implications. J Biol Chem $\mathbf{2 8 8}$ : $3500-3511$

Thomas S, Fisher KH, Snowden JA, Danson SJ, Brown S, and Zeidler MP (2015) Methotrexate is a JAK/STAT pathway inhibitor. PLoS One 10:e0130078.

Tian H and Cronstein BN (2007) Understanding the mechanisms of action of methotrexate: implications for the treatment of rheumatoid arthritis. Bull NYU Hosp Jt Dis 65:168-173.

Tolstikov V, Nikolayev A, Dong S, Zhao G, and Kuo MS (2014) Metabolomics analysis of metabolic effects of nicotinamide phosphoribosyltransferase (NAMPT) inhibition on human cancer cells. PLoS One 9:e114019.

Zimmerman MC, Clemens DL, Duryee MJ, Sarmiento C, Chiou A, Hunter CD, Tian J, Klassen LW, O'Dell JR, Thiele GM, et al. (2017) Direct antioxidant properties of methotrexate: inhibition of malondialdehyde-acetaldehyde-protein adduct formation and superoxide scavenging. Redox Biol 13:588-593.

Address correspondence to: Dr. Ryan S. Funk, Department of Pharmacy Practice, University of Kansas Medical Center, 3901 Rainbow Blvd., MS 4047, Room 6013, Kansas City, KS 66160. E-mail: ryanfunk@kumc.edu; or Rakesh K. Singh, Department of Pharmacy Practice, University of Kansas Medical Center, 3901 Rainbow Blvd., MS 4047, Room 6013, Kansas City, KS 66160. E-mail: rakeshku@kumc.edu 